\title{
Improving Teleoperation Performance in the Presence of Non-Ideal Robot Dynamics
}

\author{
M. Tavakoli, Department of Electrical and Computer Engineering, University of Alberta, Edmonton, AB, Canada \\ Robert D. Howe, School of Engineering and Applied Sciences, Harvard University, Cambridge, MA, USA \\ tavakoli@ece.ualberta.ca, howe@ seas.harvard.edu
}

\begin{abstract}
The use of thin and lightweight arms and cable-driven end-effectors in space and surgical manipulators introduces link and/or joint flexibility. When such manipulators are used as the slave robot of a master-slave teleoperation system, teleoperation performance can be degraded. We report a comparison of teleoperation system performances under slave robot link and joint flexibility (tool flexibility). We also evaluate the added benefits of using extra sensors at the tip of the flexible slave robot. It is reported that tip velocity (or position) feedback improves free-space position tracking performance in the presence of robot flexibility. Also, when the interaction forces with an environment are measured by a force sensor and fed back to the user's hand, tip velocity feedback improves hard-contact force tracking performance. During a hard contact task, tip velocity feedback can also eliminate the transmission of robot flexibility to the user's hand.
\end{abstract}

\section{INTRODUCTION}

A teleoperation system makes it possible for a user to interact with environments that are inaccessible to direct human contact, e.g., because of their constrained space or remoteness. It consists of a teleoperated robot ("slave") which interacts with an environment, and a user interface ("master") from which the human operator controls the slave. Minimally invasive surgery (MIS) and space exploration are two examples where master-slave teleoperation is advantageous. Task performance during both space and surgical teleoperation can be helped by haptic feedback [1], [2].

Current research on haptic teleoperation control assumes ideal dynamics for the slave robot, i.e., a single mass (or a mass and a damper) model for it. Such an assumption is grossly violated in current space and surgical robots due to the presence of flexibility in the links and joints of the robots. Indeed, space robots are designed to be lightweight and compact for minimum liftoff cost and energy consumption during robot control, and therefore involve flexibility. Also, surgical robots have thin instruments that enter the patient's body through small ports for minimal invasiveness and bend under contact. For instance, in the Zeus Surgical Robot System (Figure 1) from Computer Motion Inc., Goleta, CA, USA, a $1 \mathrm{~N}$ force applied to the tip of one of its cantilevered instruments (straight endo-scissors) causes a $15 \mathrm{~mm}$ tip deflection [3]. As the surgical instruments become thinner (e.g., $<3$ $\mathrm{mm}$ in pediatric surgery), the effect of tool flexibility becomes more crippling.

In addition to link flexibility, surgical robots involve joint flexibility, too. In fact, due to space limitations in MIS, actuation of a distal dexterous wrist is performed from outside

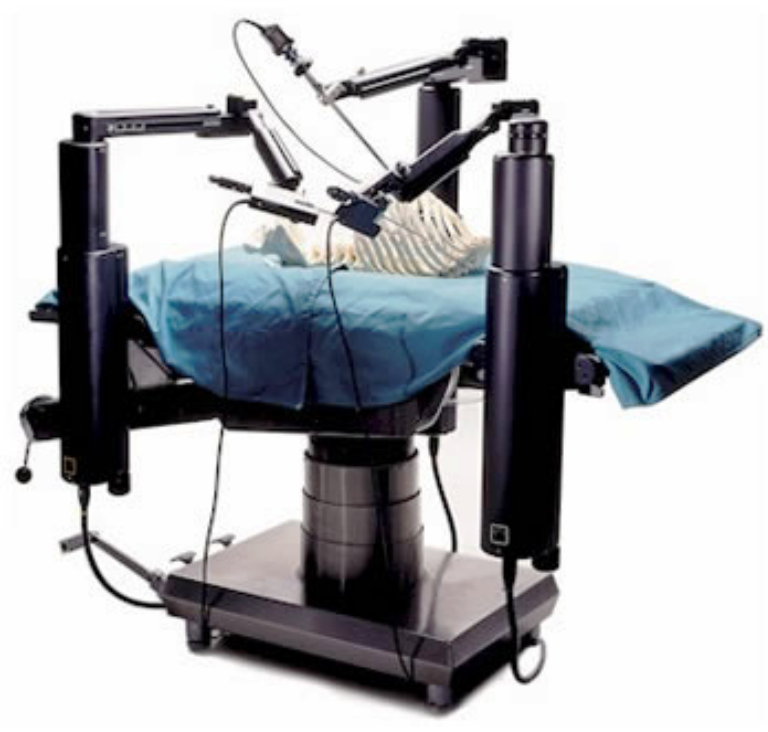

Fig. 1. The Zeus Surgical Robot System.

the patient and propagated to the wrist through flexible cables, which introduce joint flexibility. A quick solution to the problem of joint flexibility would be to increase tensions in the cable drives, which indeed minimizes joint compliance. This is the measure taken in the da Vinci Surgical System from Intuitive Surgical Inc., Sunnyvale, CA, USA. However, increased cable tension results in large friction in the instruments' drive trains and therefore impedes instrument/tissue interaction estimation for haptic feedback to the user. Given the tradeoff between joint compliance and joint friction in cable drives, joint compliance needs to be addressed separately especially in lightweight robots that cannot accommodate large actuators for overcoming drive friction.

In this research, we have systematically analyzed transparency limitations under robot link or joint flexibility and examined the added benefits offered by tip sensors during teleoperation with a flexible slave, and the cost-benefit tradeoffs of reducing or eliminating the effect of flexibility in haptic teleoperation.

\section{Model of Teleoperation System}

It can be shown that the lumped dynamics of a single flexible link are similar to the dynamics of a flexible joint consisting of a motor and an end-effector that are coupled via a finite-stiffness spring [4]. Therefore, a flexible-link slave affects teleoperation performance in the same way as a flexible-joint slave. 


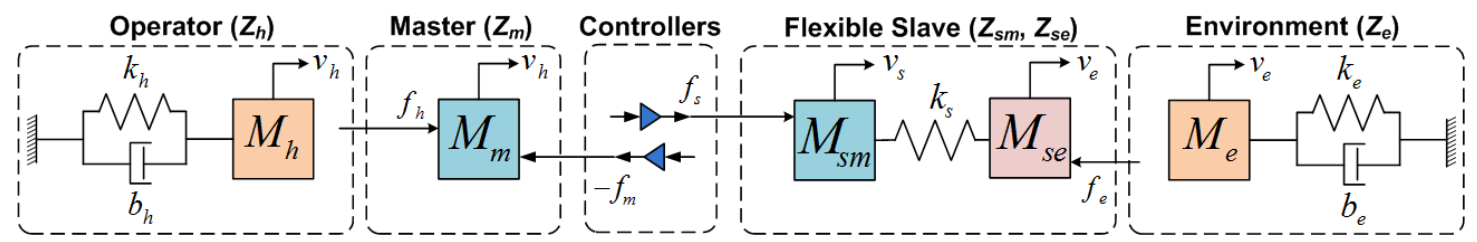

Fig. 2. Models of the operator, master, flexible slave, and environment.

The compliance in the joint of a robot can be modeled by a chained mass-spring-damper system, in which the first mass represents the joint motor whose position is measured and the last mass represents the end-effector by which the robot makes contact with the environment [5], [6]. Therefore, using the equivalent translational model of a slave with link or joint flexibility, the teleoperation system model is depicted in Figure 2. Here, the hand-master velocity is $v_{h}$ and slaveenvironment velocity is $v_{e}$. Also, $f_{h}$ and $f_{e}$ denote the forces exerted by the operator's hand on the master and by the environment on the slave, respectively. $M_{m}, M_{s}, f_{m}$ and $f_{s}$ are the master and the slave inertias and control signals, respectively. Damping terms have not been considered in the master and the slave dynamics because such terms contribute to the closed-loop equations in the same way as the derivative terms of the master and slave PD controllers, and therefore do not need to be considered separately.

The equations of motion of the flexible slave present in Figure 2 are

$$
\begin{aligned}
M_{s m} \dot{v}_{s} & =f_{s}-k_{s} \Delta x \\
M_{s i} \dot{v}_{e} & =-f_{e}+k_{s} \Delta x \\
\Delta x & =x_{s}-x_{e}
\end{aligned}
$$

where $v_{s}=\dot{x}_{s}$ and $v_{e}=\dot{x}_{e}$ are the slave's motor and end-effector velocities, respectively. The two-mass system (1)(3) has one eigenvalue at the origin of the $s$-plane and two eigenvalues at $\pm j \omega_{R}$ where

$$
\omega_{R}=\sqrt{k_{s}\left(\frac{1}{M_{s m}}+\frac{1}{M_{s e}}\right)}
$$

is the system resonance frequency. For the control input $f_{s}$, if $v_{s}$ is the output, the system will have two zeros at $\pm j \omega_{0}$ where

$$
\omega_{0}=\sqrt{\frac{k_{s}}{M_{s e}}}
$$

is the system anti-resonance frequency. If $v_{e}$ is taken as the output, however, the system will show no anti-resonant behavior.

The inertia ratio

$$
R=\frac{M_{s e}}{M_{s m}}
$$

plays a key role in shaping the dynamic characteristics of the flexible joint. When $R \ll 1$ and there is only feedback of $v_{s}$, the system shows a severely under-damped behavior [7]. In this situation, although the oscillations in $v_{s}$ may be small, those in $v_{e}$ may be large. However, with feedback of endeffector velocity $v_{e}$, it is possible to dampen such oscillations.
In the following, we examine the effect of joint (or link) flexibility in a robot that is acting as the slave during haptic teleoperation.

\section{EFFECT OF Robot FleXibility on Teleoperation PERFORMANCE}

In an ideally transparent teleoperation system [8], through appropriate control outputs $f_{m}$ and $f_{s}$, the positions and contact forces at the master and the slave ends will match regardless of the operator and environment dynamics

$$
v_{h}=v_{e}, \quad f_{h}=f_{e}
$$

Condition (7) guarantees that the dynamics of the environment is displayed to the user with no distortion. We consider the following elements of hybrid, transmission and impedance matrices (in the $s$-domain) as our transparency indices:

- Free-motion transmitted impedance $F_{h} /\left.V_{h}\right|_{F_{e}=0}$,

- Free-motion position tracking index $-V_{e} /\left.V_{h}\right|_{F_{e}=0}$,

- Hard-contact transmitted impedance index $F_{h} /\left.F_{e}\right|_{V_{e}=0}$,

- Hard-contact transmitted impedance $F_{h} /\left.V_{h}\right|_{V_{e}=0}$.

We consider two teleoperation control architectures that are most commonly used due to their implementation simplicity. Position error based (PEB) bilateral control uses no force sensor measurements and, using PD position controllers on the master and the slave sides, merely tries to minimize the difference between the master and the slave positions for providing haptic feedback to the user. Direct force reflection (DFR) bilateral control uses a PD position controller to ensure that the slave follows the position of the master, however it employs a force sensor to directly measure slave-environment interactions for reflecting them to the user. For both teleoperation control methods, we examined the effect of position and/or force sensors at the tip of a flexible slave on the above four performance metrics across the whole frequency range and investigated the effect on the bandwidths of position and force tracking indexes.

\section{Main Results And Discussion}

The questions addressed in this research were, what are the limitations imposed by the slave robot flexibility on teleoperation transparency (as defined by the aforementioned four performance metrics), what added benefits can tip sensors deliver during teleoperation with a flexible slave, and what are the cost-benefit tradeoffs of reducing or eliminating the effect of flexibility in haptic teleoperation? While readers are referred to [9], [4] for the complete analysis, below are the main results: 
- When the slave is in free space, unlike DFR teleoperation in which the user only feels the master inertia, in PEB teleoperation the user feels an additional impedance (which can be large). Such a residual impedance can create problems in terms of detecting small contacts or contact with very soft tissue, and using a force sensor at the slave helps to avoid it.

- For both PEB and DFR teleoperation architectures and at high frequencies, velocity (or position) feedback from the tip of the flexible slave improves free-space position tracking performance, which is otherwise hampered by the anti-resonance of the two-mass-spring model. It is of practical interest to maintain good position tracking bandwidth in order to enable accurate and fast manipulation.

- In DFR teleoperation and at high frequencies, tip velocity feedback improves hard-contact force tracking performance. Without tip velocity feedback, force tracking response will be band-limited, and the system will not be able to accurately simulate high-frequency haptic phenomena such as edges or surface texture of an object.

- For both PEB and DFR teleoperation architectures and over low frequencies, free-space position tracking and hard-contact force tracking are both satisfactory even in the absence of tip velocity feedback. However, in terms of the transmitted impedance, it was found that the only way to eliminate the display of robot flexibility to the user (even over low frequencies) is to employ tip velocity feedback. Previously, Christiansson and van der Helm [10] had concluded through experimental measurements with a low-stiffness slave that the maximum transmitted impedance can be doubled if tip velocity feedback is used in a more sophisticated "4-channel" bilateral teleoperation architecture. Consistent with their results, we found that tip velocity feedback helps achieve an infinitely stiff transmitted impedance in theory even with PEB and DFR teleoperation architectures, which have lower implementation complexity. The significance of this result is in the fact that if the robot flexibility is transmitted to the user, it will limit the perception of hitting a hard object (such as bone) and the usefulness of haptic cues for soft-tissue stiffness discrimination. This has direct consequences, for example, in tissue palpation as a means to detect cancerous tissue, which has a different stiffness compared to healthy tissue.

\section{CONCluding Remarks}

This paper discussed the limitations imposed by the slave robot link or joint flexibility on master-slave teleoperation transparency and how extra sensors at the end-effector (tip) of the flexible slave robot would benefit transparency. Here is a summary of the main results: First, tip velocity (or position) feedback improves free-space position tracking under robot flexibility. Second, depending on which teleoperation control method is used, tip velocity feedback may improve hardcontact force tracking performance. Third, during a hard contact task, tip velocity feedback can eliminate the transmission of robot flexibility to the user's hand.

\section{ACKNOWLEDGEMENTS}

This research was supported by the NSF grant EEC-9731748 and an NSERC Postdoctoral Fellowship awarded to M. Tavakoli.

\section{REFERENCES}

[1] T. Imaida, Y. Yokokohji, T. Doi, M. Oda, and T. Yoshikawa, "Groundspace bilateral teleoperation of ETS-VII robot arm by direct bilateral coupling under 7-s time delay condition," IEEE Transactions on Robotics and Automation, vol. 20, no. 3, pp. 499-511, 2004.

[2] C. Wagner and R. Howe, "Force feedback benefit depends on experience in multiple degree of freedom robotic surgery task," IEEE Transactions on Robotics, vol. 23, no. 6, pp. 1235-1240, 2007.

[3] R. A. Beasley and R. D. Howe, "Model-based error correction for flexible robotic surgical instruments," in Proceedings of Robotics: Science and Systems Conference, Cambridge, MA, June 2005, pp. 359-364.

[4] M. Tavakoli and R. D. Howe, "Haptic implications of tool flexibility in surgical teleoperation," in Proceedings of 2008 Symposium on Haptic Interfaces for Virtual Environments and Teleoperator Systems, Reno, NV, March 2008, pp. 377-388.

[5] M. W. Spong, "Modeling and control of elastic joint robots," ASME Journal of Dynamic Systems, Measurement and Control, vol. 109, no. 4, pp. 310-319, 1987.

[6] J. K. Mills, "Stability and control of elastic-joint robotic manipulators during constrained-motion tasks," IEEE Transactions on Robotics, vol. 8, no. 1, pp. 119-126, February 1992.

[7] G. Zhang and J. Furusho, "Speed control of two-inertia system by PI/PID control," IEEE Transactions on Industrial Electronics, vol. 47, no. 3, pp. 603-609, June 2000.

[8] B. Hannaford, "A design framework for teleoperators with kinesthetic feedback," IEEE Transactions on Robotics and Automation, vol. 5, pp. 426-434, 1989.

[9] M. Tavakoli and R. D. Howe, "The effect of joint elasticity on bilateral teleoperation," in Proceedings of 2007 International Conference on Intelligent Robots and Systems, San Diego, CA, October 2007, pp. 16181623.

[10] G. A. V. Christiansson and F. C. T. van der Helm, "The low-stiffness teleoperator slave - a trade-off between stability and performance," The International Journal of Robotics Research, vol. 26, no. 3, pp. 287-299, March 2007. 